“C 2007 IEEE. Personal use of this material is permitted. Permission from IEEE must be obtained for all other uses, in any current or future media, including reprinting/republishing this material for advertising or promotional purposes, creating new collective works, for resale or redistribution to servers or lists, or reuse of any copyrighted component of this work in other works." 


\title{
Advanced robust tracking control of a powered wheelchair system
}

\author{
Nghia T. Nguyen, Hung T. Nguyen, Senior Member, IEEE and Steven Su, Member, IEEE
}

\begin{abstract}
In this paper, the dynamic multivariable model of the wheelchair system is obtained including the presence of transportation lags. The triangular diagonal dominance (TDD) decoupling technique is applied to reduce this multivariable control problem into two independent scalar control problems. An advanced robust control technique for the wheelchair has been developed based on the combination of a TDD decoupling strategy and neural network controller design. The results obtained from the real-time implementation confirm that robust performance for this multivariable wheelchair control system can indeed be achieved.
\end{abstract}

\section{INTRODUCTION}

$\mathrm{W}$ HEELCHAIRS as mobility aids to the disabled and elderly are increasing important due to the increase in ageing population and accidents. It is important to offer users smooth and safe driving conditions with a quick response. In order to achieve this, the control system should be able to respond to the user's commands in the shortest possible time and with greatest accuracy. In addition, under various conditions, a powered wheelchair system can be regarded as a nonlinear multivariable system [1].

In literature, various advanced control strategies for powered wheelchair applications have been developed: an adaptable optimal controller [2], digital controller [3], advanced digital control techniques [4] and neural control technique [5]. However, none of these techniques have treated powered wheelchairs as a multivariable system and as a consequence, it is expected that the above control strategies would not be optimal when the operating environment for the wheelchairs is rough and nonlinear.

Regarding a wheelchair as a multivariable system, decoupling control techniques can be developed in order to decompose the wheelchair system into two scalar systems which can be designed independently. Various decoupling control techniques have been proposed [6-9], but these techniques are only applicable for linear systems.

Equipped with many desirable properties such as learning by experience, ability to generalise and map nonlinear functions, robustness in the presence of noise and multivariable interactions, neural network control systems have provided effective solutions for complex and nonlinear

This work was supported in part by Australian Research Council under Discovery Grant DP0666942 and LIEF Grant LE0668541.

Nghia T. Nguyen is with Faculty of Engineering, University of Technology, Sydney, Broadway, NSW 2007, Australia (phone: +612-95142451; fax: +61 29514 2868; e-mail: tnnguyen@eng.uts.edu.au).

Hung .T. Nguyen is with Faculty of Engineering, University of Technology, Sydney, Broadway, NSW 2007, Australia (e-mail: Hung.Nguyen@uts.edu.au).

Steven $\mathrm{Su}$ is with Faculty of Engineering, University of Technology, Sydney, Broadway, NSW 2007, Australia (e-mail: Steven.Su@uts.edu.au). control problems with and without uncertainties [10-12].

As neural network controllers can be developed to provide robust solutions to nonlinear multivariable control problems, we aim to develop a novel control structure applicable for powered wheelchair systems by combining neural network controllers and decoupling techniques.

The paper is organised as follows. In the next section, the dynamic multivariable model of a powered wheelchair system is obtained and developed experimentally. A conventional decoupling control method is presented in Section 3. An advanced robust control based combination of TDD and neural network is presented in Section 4. Finally, a conclusion can be found in Section 5 .

\section{MODELLING OF A POWERED WHEELCHAIR SYSTEM}

The wheelchair system as shown in Fig. 1 has two rear driving wheels and two free caster front ones. The drive system associated with each active wheel is composed of a DC motor, and the wheelchair is fitted with an incremental optical encoder and a gear-head. The power for the drive system is supplied by a $24 \mathrm{~V}$ battery. A personal computer attached at the back of the wheelchair plays the role of an intelligent controller for this system.

As the powered wheelchair system is a multivariable system, its dynamic model can be presented below.

$$
\left[\begin{array}{l}
v(s) \\
\omega(s)
\end{array}\right]=\left[\begin{array}{ll}
g_{11}(s) & g_{12}(s) \\
g_{21}(s) & g_{22}(s)
\end{array}\right]\left[\begin{array}{l}
u_{1}(s) \\
u_{2}(s)
\end{array}\right]
$$

where $u=\left[u_{1}(s) u_{2}(s)\right]^{T}$ is the actuator voltage input vector, v(s) and $\omega(s)$ are the linear output velocity and the angular output velocity respectively.

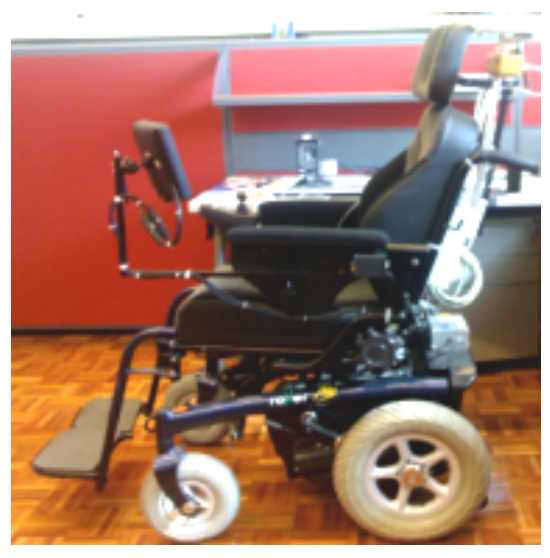

Fig. 1: A powered wheelchair 
As servo systems, the elements of dynamic model of the wheelchair can be written in the form.

$$
g_{i j}(s)=\frac{K_{i j}}{\left(1+s T_{i j}\right)} e^{-\tau_{i j}} ; i, j=1,2
$$

Introducing the step inputs ranged from a minimum value of $-1 \mathrm{~V}$ to a maximum value of $1 \mathrm{~V}$, the corresponding output responses for this system can be obtained.

An uncertain multivariable model for this system is developed by applying inputs (ranged between $-1 \mathrm{~V}$ and $1 \mathrm{~V}$ ) to the two DC motors and 80 step responses are collected overall. As a result, three dynamic models of the wheelchair system are obtained. These dynamic models correspond to the nominal transfer matrix $\mathrm{G}_{0}(\mathrm{~s})$, the upper-bounded transfer matrix $\mathrm{G}_{1}(\mathrm{~s})$ and the lower-bounded transfer matrix $\mathrm{G}_{2}(\mathrm{~s})$ as shown in Equations (3), (4), and (5):

$$
\begin{aligned}
& G_{0}(s)=\left[\begin{array}{ll}
\frac{1.4}{(1+0.8 s)} e^{-0.22 s s} & \frac{0.125}{(1+0.4 s)} e^{-0.15 s} \\
\frac{0.1}{(1+0.35 s)} e^{-0.2 s} & \frac{1.8}{(1+0.5 s)} e^{-0.2 s}
\end{array}\right] . \\
& G_{1}(s)=\left[\begin{array}{ll}
\frac{1.8}{(1+0.55 s)} e^{-0.1 s} & \frac{0.22}{(1+0.35 s)} e^{-0.1 s} \\
\frac{0.25}{(1+0.3 s)} e^{-0.1 s} & \frac{2.6}{(1+0.45 s)} e^{-0.1 s}
\end{array}\right] \\
& G_{2}(s)=\left[\begin{array}{ll}
\frac{1}{(1+1.05 s)} e^{-0.35 s} & \frac{0.04}{(1+0.45 s)} e^{-0.2 s} \\
\frac{0.005}{(1+0.4 s)} e^{-0.3 s} & \frac{1}{(1+0.55 s)} e^{-0.3 s}
\end{array}\right]
\end{aligned}
$$

As the transportation lag terms can be approximated as the first order as follows.

$$
e^{-\tau s}=\frac{1}{1+\tau s}
$$

the nominal model of the wheelchair system $\mathrm{G}_{0}(\mathrm{~s})$ can be simplified to:

$$
G_{0}(s)=\left[\begin{array}{cc}
\frac{1.4}{(1+0.8 s)(1+0.225 s)} & \frac{0.125}{(1+0.4 s)(1+0.15 s)} \\
\frac{0.1}{(1+0.35 s)(1+0.2 s)} & \frac{1.8}{(1+0.5 s)(1+0.2 s)}
\end{array}\right]
$$

\section{DECOUPLING CONTROL OF THE WHEELCHAIR SYSTEM}

\section{A. Decoupling design of the wheelchair system}

As the powered wheelchair system is an uncertain multivariable system, to simplify the control design procedure, the overall system can be decomposed into two independent scalar control systems using a decoupler $\mathrm{D}(\mathrm{s})$ as shown in Fig. 2.

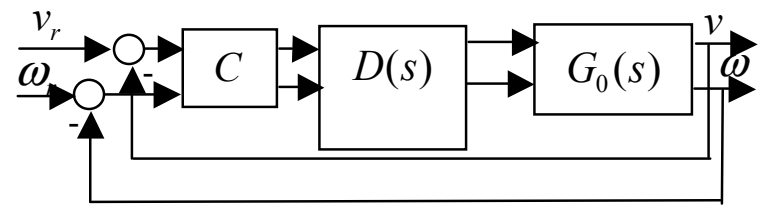

Figure 2: Decoupled control structure of the wheelchair system
Using the triangularisation technique [9], the following steps are made in order to construct the desirable decoupler $\mathrm{D}(\mathrm{s})$ :

Step 1: In the first row, the element $(1,1)$ already has the lowest degree $(\delta=2)$.

Step 2: Subtract a multiple of the first column from the

$$
\frac{0.1 .25}{(1+0.4 s)(1+0.15 s)}=a\left(\frac{1.4}{(1+0.8 s)(1+0.225 s)}\right)+b
$$

Where:

$$
a=\left(\frac{0.125}{1.4}\right)\left(\frac{(1+0.8 s)(1+0.225 s)}{(1+0.4 s)(1+0.15 s)}\right) \text { and } \mathrm{b}=0
$$

Thus compensator is chosen as.

$$
D(s)=\left[\begin{array}{cc}
1 & -\left(\frac{0.125}{1.4}\right)\left(\frac{(1+0.8 s)(1+0.225 s)}{(1+0.4 s)(1+0.15 s)}\right) \\
0 & 1
\end{array}\right]
$$

Therefore, the decoupled transfer function matrix

$$
\begin{aligned}
& P_{0}(s)=G_{0}(s) \cdot D(s)= \\
& {\left[\begin{array}{lc}
\frac{280}{(5+4 s)(40+9 s)} & 0 \\
\frac{10}{(20+7 s)(5+s)} & \frac{41436(\mathrm{~s}+6.74)(\mathrm{s}+2.89)(\mathrm{s}+2.48)}{56(20+7 \mathrm{~s})(5+s)(5+2 s)(20+3 s)(2+s)}
\end{array}\right]}
\end{aligned}
$$

The obtained decoupled transfer function matrix is triangular-diagonal-dominant (TDD). The nominal transfer function matrix can be simplified further as shown below:

$$
P_{0}(s)=\left[\begin{array}{cc}
\frac{280}{(5+4 s)(40+9 s)} & 0 \\
\frac{10}{(20+7 s)(5+s)} & \frac{123.32(s+2.89)}{(20+7 \mathrm{~s})(5+s)(2+s)}
\end{array}\right]
$$

\section{B. Controller design of the wheelchair system}

As the decoupled system Go(s)D(s) is triangular-diagonaldominant, only the two diagonal elements $\operatorname{Po}(1,1)$ and $\operatorname{Po}(2,2)$ are required for controller design. Root locus techniques are then used to design two independent controllers to satisfy the second-order specification with a settling time $\mathrm{T}_{\mathrm{s}} \leq 4 \mathrm{~s}$, a peak overshoot to a step input $\mathrm{M}_{\mathrm{p}} \leq$ $5 \%$ and zero steady error to a step input.

The following controller transfer function matrix has been designed to satisfy the above performance specifications.

$$
C(s)=\left[\begin{array}{cc}
0.25\left(\frac{s+2.5}{s}\right) & 0 \\
0 & 0.3\left(\frac{s+3}{s}\right)
\end{array}\right]
$$

\section{Results}

The simulation result in Figure 3 shows the responses of various models using obtained decoupler $\mathrm{D}(\mathrm{s})$ and controller $\mathrm{C}(\mathrm{s})$. 


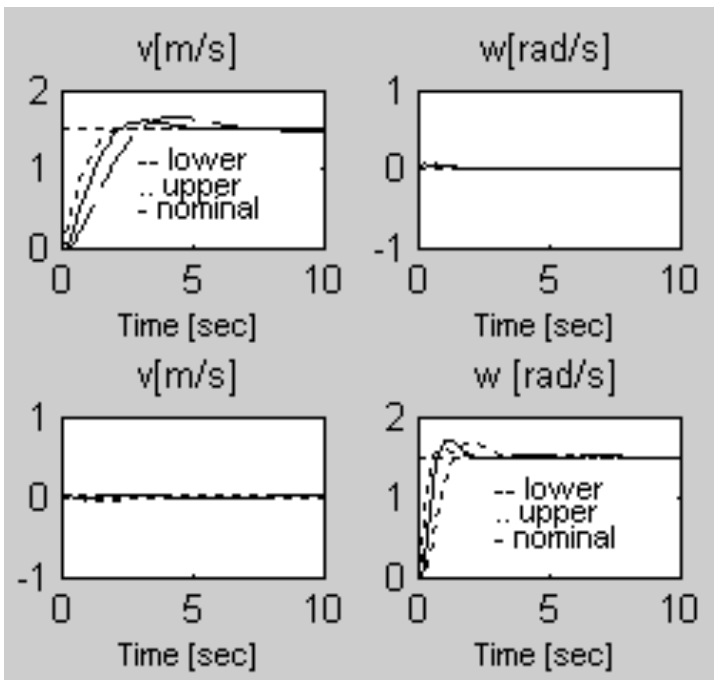

Figure 3: Conventional controller responses-simulation results

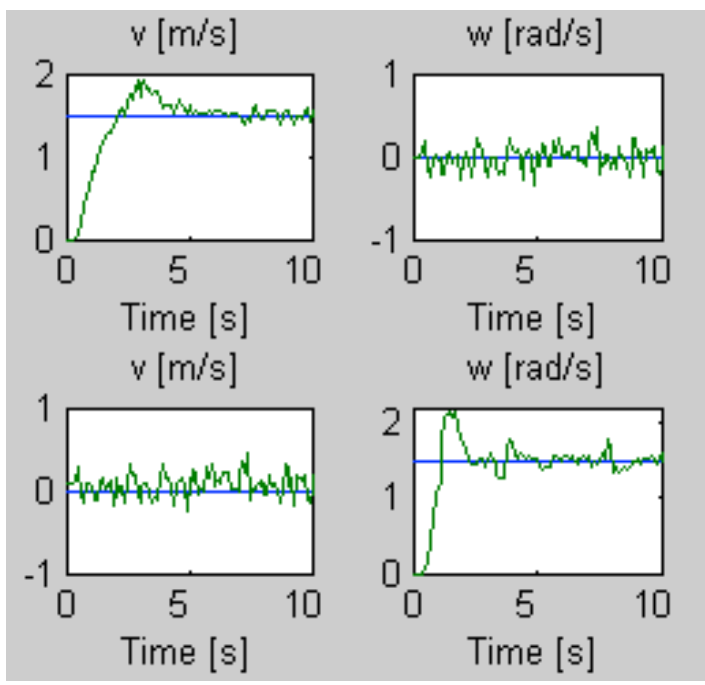

Figure 4: Conventional controller responses-practical results

To validate the performance of this design, real-time implementation is conducted. The algorithms are written in $\mathrm{C}++$ using Visual $\mathrm{C}++$ software. The sampling time $\mathrm{Ts}$ is chosen as 100 [ms]. The result is shown on Figure 4.

\section{ADVANCED ROBUST CONTROL BASED ON NEURAL NETWORK OF THE WHEELCHAIR SYSTEM}

\section{A. Advanced robust control structure}

The conventional controllers are synthesised under an assumption of neglecting the effect of the element $(2,1)$ in the equation (10). This is a valid design procedure because the decoupled system is TDD. However, as the overall model of the wheelchair may vary from the lower-bounded transfer function matrix to the upper-bounded transfer function matrix, the system performance may not be guaranteed.

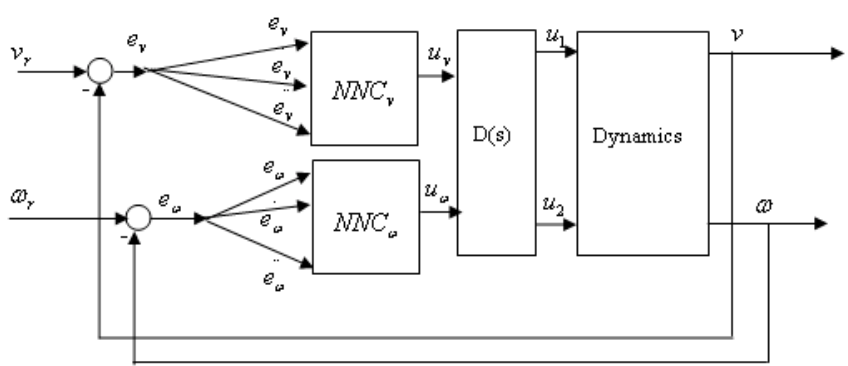

Figure 5: Neural control scheme of the wheelchair system

In order to improve the robustness of the system regardless of the changes in the wheelchair model, a neural network controller can be developed to replace the convention one

The proposed neural controller scheme for the wheelchair system is illustrated in Figure 5.

Define $f_{1}(.) ; f_{2}($.$) is the activation function of hidden$ layer and output layer respectively. For a given structure of the neural network controller, the output of the neural network can be calculated as follows:

$$
u=\sum_{i=1}^{m} f_{2}\left(w_{i} \sum_{j=1}^{n} f_{1}\left(v_{i j} e_{j}\right)\right)
$$

Define the objective function as following:

$$
E=\frac{1}{2}\left(y_{d}-y\right)^{2}
$$

To minimise $\mathrm{E}$, it is necessary to change the weights of neural network in the direction of negative gradient.

$$
\begin{gathered}
\Delta w_{i}=-\alpha \frac{\partial E}{\partial w_{i}}=-\alpha \frac{\partial E}{\partial y} \frac{\partial y}{\partial u} \frac{\partial u}{\partial w_{i}} \\
\Delta v_{i j}=-\alpha \frac{\partial E}{\partial v_{i j}}=-\alpha \frac{\partial E}{\partial y} \frac{\partial y}{\partial u} \frac{\partial u}{\partial v_{i j}} \\
\frac{\partial u}{\partial w_{i}}=f_{2}^{\prime}\left(\sum_{i=1}^{m} w_{i} f_{1}\left(\sum_{j=1}^{n} v_{i j} e_{j}\right)\right) f_{1}\left(\sum_{j=1}^{n} v_{i j} e_{j}\right) \\
\frac{\partial u}{\partial v_{i j}}=f_{2}^{\prime}\left(\sum_{i=1}^{m} w_{i} f_{1}\left(\sum_{j=1}^{n} v_{i j} e_{j}\right)\right) f_{1}^{\prime}\left(\sum_{j=1}^{n} v_{i j} e_{j}\right) w_{i} e_{j}
\end{gathered}
$$

In order to train the neural networks, the plant Jacobian matrix can be calculated from the nominal model of the wheelchair system. The update rules are:

$$
\begin{aligned}
& w_{i}(k)=w_{i}(k-1)+\Delta w_{i}(k) \\
& v_{i j}(k)=v_{i j}(k-1)+\Delta v_{i j}(k)
\end{aligned}
$$

\section{B. Results}

Optimal neural controllers have been trained to meet the second-order specification with settling time $T_{\mathrm{s}} \leq 4(\mathrm{~s})$, peak overshoot to a step input $\mathrm{M}_{\mathrm{p}} \leq 5 \%$, and zero steady error to a step input. The structure of the $N N C_{v}$ is chosen as $\{3,4,1\}$, which is equivalent to 3 input nodes, 4 hidden nodes and a output node, whereas the structure of $N N C_{\omega}$ is $\{3,5,1\}$. 
Convert the two element $\mathrm{P}_{0}(1,1)$ and $\mathrm{P}_{0}(2,2)$ into discrete form with sampling time as $100[\mathrm{~ms}]$, we obtain as follows:

$v(t)=0.033 u_{v}(t)+0.027 u_{v}(t-1)+1.53 v(t-1)-0.56 v(t-2)$

$\omega(t)=0.07 u_{\omega}(t)+0.056 u_{\omega}(t-1)+1.42 \omega(t-1)-0.49 \omega(t-2)$

Therefore, the plant Jacobian matrix is calculated as:

$$
J(t)=\left[\begin{array}{cc}
\frac{\partial v(t)}{\partial u_{v}(t)} & 0 \\
0 & \frac{\partial \omega(t)}{\partial u_{\omega}(t)}
\end{array}\right]=\left[\begin{array}{cc}
0.033 & 0 \\
0 & 0.07
\end{array}\right]
$$

The sigmoid and linear activation functions are used for hidden and output layer respectively for both neural controllers with a learning rate of 0.015 . Figure 6 shows the robustness of advanced controllers based on combination of TDD and neural controllers.

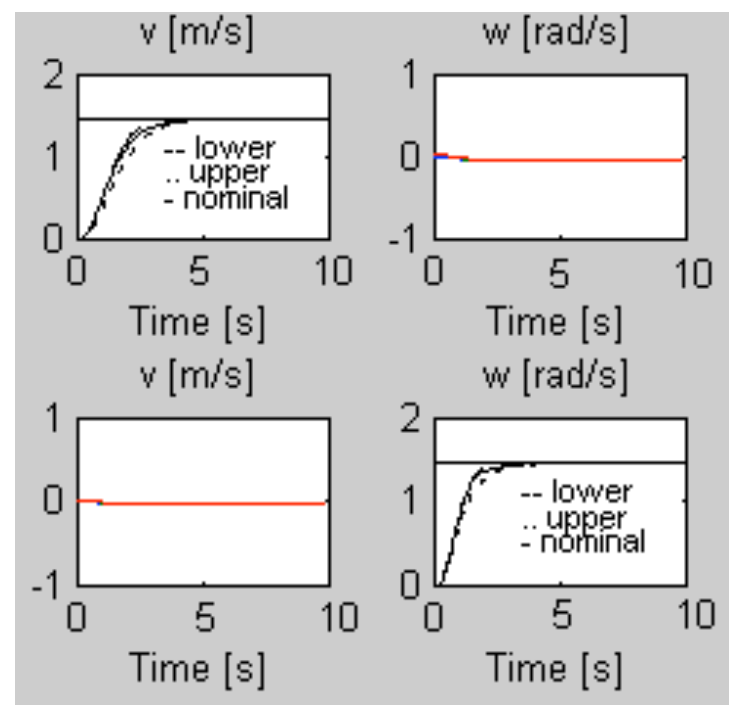

Figure 6: Robust neural controller responses - simulation results

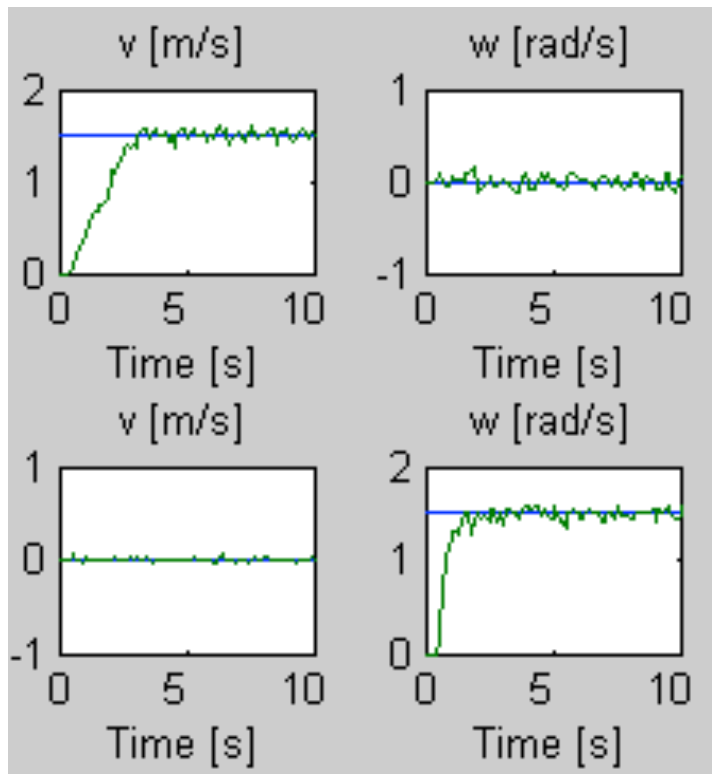

Figure 7: Robust neural controller responses - practical results
To validate the method, two real-time experiments are presented. The first one we put reference input $v_{r}=1.5(\mathrm{~m} / \mathrm{s})$ while keeping $\omega_{r}=0(\mathrm{rad} / \mathrm{s})$. Then we put $v_{r}=0(\mathrm{~m} / \mathrm{s})$ and $\omega_{r}=1.5(\mathrm{rad} / \mathrm{s})$. The results are shown in Figure 7 .

\section{CONCLUSION}

In this paper, we have presented a new control structure by combining the decoupling control technique with neural network controllers for the powered wheelchair system. This multivariable system is decoupled into two independent subsystems using the TDD decoupling technique, and the two neural network controllers are designed and implemented with the aim to improve the robustness and control performance of this system. The results obtained from the real-time implementation confirm that robust performance for this multivariable wheelchair control system can indeed be achieved.

\section{REFERENCES}

[1] D. Ding and R. A. Cooper, "Electric-powered wheelchairs - a review of current technology and insight into future directions," in IEEE Control Syst Magazine, vol. 25, April 2005, pp. 22-34.

[2] K. E. Brown., R. M. Inigo, and B. W. Johnson, "Design, Implementation, and Testing of an Adaptable Optimal Controller for an Electric Wheelchair," IEEE Trans on Industry Application, vol. 26, pp. 1144-1157, Dec 1990.

[3] R.-X. Chen, L.-G. Chen, and L. Chen, "System Design Consideration for Digital Wheelchair controller," IEEE Trans on Industrial Electronics, vol. 47, pp. 898-907, Aug 2000.

[4] F. Espinosa, E. Lopez, R. Mateos, M. Mazo, and R. Garcia "Application of advanced digital control techniques to the drive andtrajectory tracking system of a wheelchair for the disabled," IEEE Inter Conf on Emerging Technologies and Factory Automation, vol. 1, pp. 521-528, 1999.

[5] L. Boquete, R. Barea, R. Garca, M. Mazo, and F. Espinosa, "Identification and control of a wheelchair using recurrent neural networks " Eng Appl of Artificial Inte vol. 12, pp. 443-452, 1999.

[6] S. R. Weller and G. C. Goodwin, "Controller design for partial decoupling of linear multivariable systems," Proceedings of the IEEE 32nd Conf on Decision and Control, pp. 833-834, 1993.

[7] C. Desoer and A. N. Gundes, "Decoupling linear multiinput multioutput plants by dynamic output feedback: An algebraic theory," IEEE Trans. on Auto Control, vol. 31, pp. 744- 750, 1986.

[8] S. Singh, "Decoupling of invertible nonlinear systems with state feedback and precompensation," IEEE Transactions on Automatic Control, vol. 25, pp. 1237-1239, Dec 1980.

[9] N. Hung and B. Anderson, "Triangularization technique for the design of multivariable control systems," IEEE Transactions on Automatic Control, vol. 24, pp. 455-460, 1979.

[10] K. S. Narendra and K. Parthasarathy, "Identification and control of dynamical systems using neural networks," IEEE Trans on Neural Networks, vol. 1, pp. 4--27, 1990.

[11] V. C. Chen and Y.-H. Pao, "Learning control with neural networks," presented at IEEE International Conference on Robotics and Automation, 1989.

[12] D. Psaltis, A. Sideris, and A. A. Yamamura, "A multilayered neural network controller," IEEE Control Systems Magazine, vol. 8, pp. 17$21,1988$. 